\title{
Measurements of the top quark mass with the ATLAS detector
}

\section{Richard Nisius ${ }^{* \dagger}$}

Max-Planck-Institut für Physik (Werner-Heisenberg-Institut) Föhringer Ring 6, D-80805

München, Germany

E-mail: Richard.Nisiusempp.mpg.de

The measurements of the top quark mass given are obtained from ATLAS data taken at protonproton centre-of-mass energies of $\sqrt{s}=7$ and $8 \mathrm{TeV}$. An extraction of the top quark pole mass $\left(m_{\text {top }}^{\text {pole }}\right)$ at next-to-leading order $(\mathrm{NLO})$ is presented. This result is obtained from normalised differential cross-sections in the $t \bar{t} \rightarrow$ dilepton channel leading to: $m_{\text {top }}^{\text {pole }}=173.2 \pm$ 0.9 (stat.) \pm 0.8 (syst.) \pm 1.2 (theo.) GeV. In addition, measurements of $m_{\text {top }}$ are discussed that are based on the template method performed in three $t \bar{t}$ decay channels. For all results the uncertainty is dominated by systematic effects. Finally, the 2016 ATLAS combined value of $m_{\text {top }}$ is: $m_{\text {top }}=172.84 \pm 0.34$ (stat.) \pm 0.61 (syst.) $\mathrm{GeV}$, with a total uncertainty of $0.70 \mathrm{GeV}$, i.e. a precision of $0.4 \%$.

The European Physical Society Conference on High Energy Physics

5-12 July, 2017

Venice

*Speaker.

$\dagger$ On behalf of the ATLAS Collaboration 


\section{Introduction}

The Large Hadron Collider (LHC) is a top quark ${ }^{1}$ factory. The largest rate of events with top quarks is obtained from $t \bar{t}$ production. The $t \bar{t}$ decay channels are classified by the $W^{+} W^{-}$decays and are named the $t \bar{t} \rightarrow$ dilepton, $t \bar{t} \rightarrow$ lepton+jets and $t \bar{t} \rightarrow$ all-jets channels.

The mass of the top quark $\left(m_{\mathrm{top}}\right)$ is a fundamental parameter of the Standard Model (SM) of particle physics. To obtain $m_{\text {top }}$ from data, two conceptually different approaches are followed. Firstly, as has been done since the discovery of the top quark in 1995, $m_{\text {top }}$ is measured from $t \bar{t}$ final state objects by means of template analyses. The templates are obtained from Monte Carlo (MC) simulated events using different assumed values for the top quark mass parameter in the program. Therefore, measurements of $m_{\text {top }}$ obtained with this method relate to measurements of the input parameter of MC programs, i.e. not to a specific program, since the differences of the programs used, are covered by the systematic uncertainty. Secondly, in recent years also $m_{\text {top }}^{\text {pole }}$ is extracted based on experimental quantities that are corrected for detector effects and compared to perturbative calculations at next-to-leading order (NLO) in perturbative QCD, performed in a well-defined renormalisation scheme. Presently, the two attempts are complementary. While results of the first type are more precise, those of the second relate to a more accurate theoretical definition of the top quark mass. The relation between $m_{\mathrm{top}}^{\text {pole }}$ and $m_{\text {top }}$ is a matter of theoretical debate. Once this issue is resolved, analyses leading to the more precise results will be preferred.

The details of the ATLAS [1] analyses presented here are given in the respective publications $[2,3,4,5]$. In this short write-up, only the main aspects of the analyses are discussed.

\section{Determination of the top quark pole mass}

Because measurements of $m_{\mathrm{top}}$ are mostly limited by systematic uncertainties related to the hadronic final state, purely leptonic variables have been advertised for measurements of the top quark mass. The extraction [2] of $m_{\text {top }}^{\text {pole }}$ is performed in the $t \bar{t} \rightarrow e \mu+X$ channel, using normalised lepton differential cross-sections, $\frac{1}{\sigma_{x}} \frac{d \sigma_{x}}{d x}$ for five variables, $x=p_{\mathrm{T}}^{\ell}, p_{\mathrm{T}}^{e \mu}, m^{e \mu}, p_{\mathrm{T}}^{e}+p_{\mathrm{T}}^{\mu}$ and $E^{e}+E^{\mu}$. One example distribution is shown in Fig. 1(a). Subsequently, the distributions are background subtracted, corrected to stable particle level using Powheg+Pythia6+CT10 as explained in Ref. [2], and finally normalised to unity. A clear sensitivity to the top quark mass is observed for all five variables. A comparison to a fixed order prediction at NLO in production and decay is shown in Fig. 1(b), where the bars denote the full data uncertainty. Within the sizeable uncertainties, the fixed order prediction describes the data. The measurements of $m_{\text {top }}^{\text {pole }}$ in Fig. 1(c) are obtained from fits to individual differential cross-sections, or to all, including those for $\left|\eta^{\ell}\right|,\left|y^{e \mu}\right|, \Delta \Phi^{e \mu}$. The latter distributions are not sensitive to $m_{\text {top }}^{\text {pole }}$, but help constrain the PDF and the renormalisation and factorisation scale variation induced uncertainties in $m_{\text {top }}^{\text {pole }}$. The spread in the individual results of $m_{\text {top }}^{\text {pole }}$ is about $6 \mathrm{GeV}$. The combined result is: $m_{\mathrm{top}}^{\text {pole }}=173.2 \pm 0.9$ (stat.) \pm 0.8 (syst.) \pm 1.2 (theo.) $\mathrm{GeV}=$ $173.2 \pm 1.6 \mathrm{GeV}$. The theoretical uncertainty originates from PDF $(0.3 \mathrm{GeV})$ and scale variations $(1.1 . \mathrm{GeV})$, the latter obtained from variations using either fixed or dynamic (e.g. $E_{\mathrm{T}} / 2$ ) scales. The first NLO extraction of $m_{\text {top }}^{\text {pole }}$ using this method results in an uncertainty of $1.6 \mathrm{GeV}$, to be compared with the precision of the results presented in the next section.

\footnotetext{
${ }^{1}$ Charge conjugation is implied throughout and natural units are used, $c=\hbar=1$.
} 


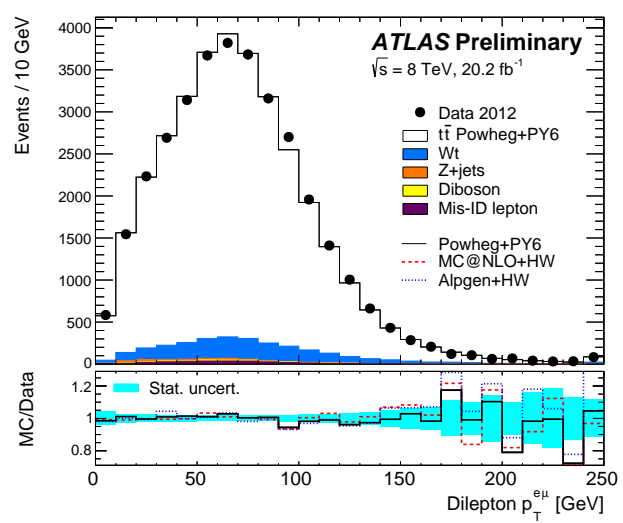

(a) Data versus MC simulation

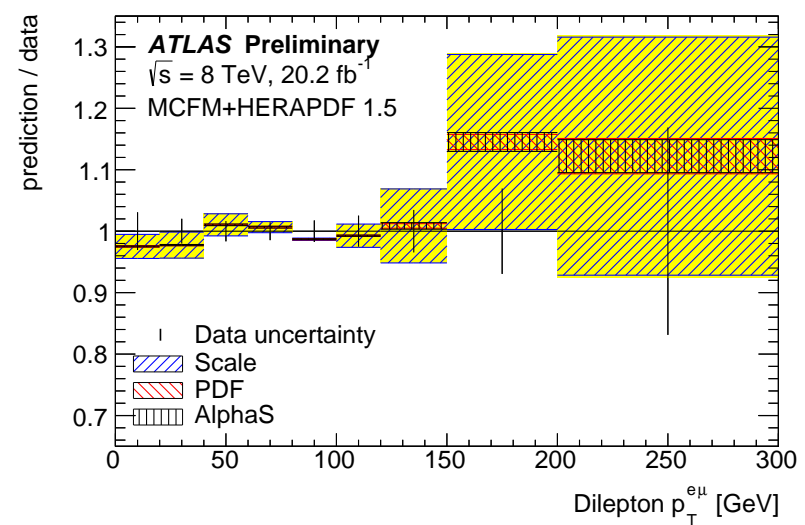

(b) Data versus NLO MCFM prediction

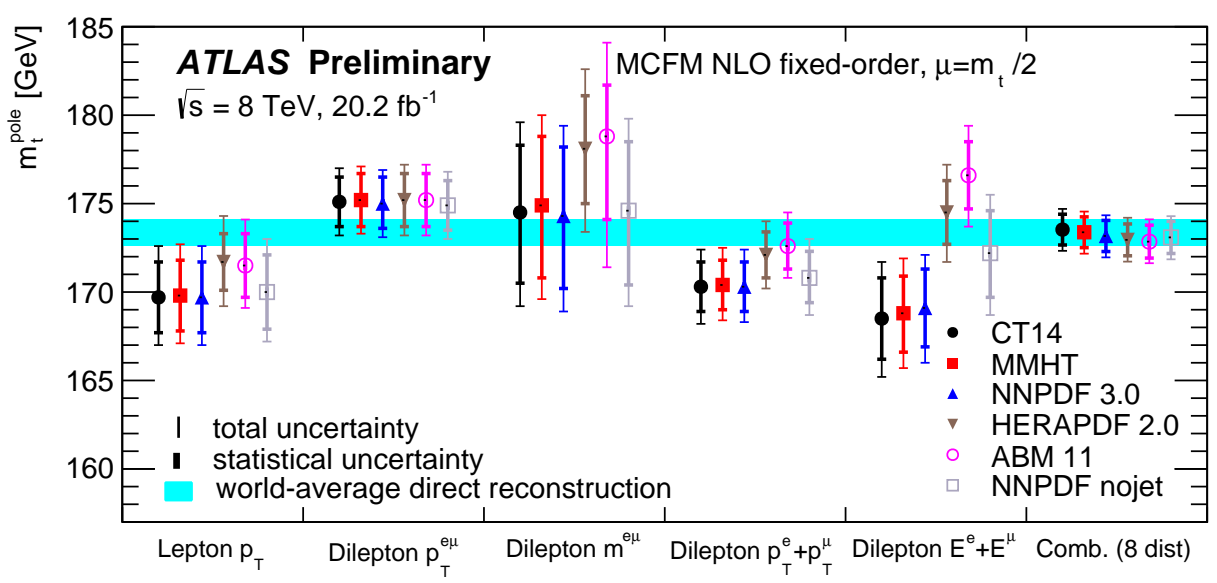

(c) Fitted values of $m_{\text {top }}^{\text {pole }}$

Figure 1: Extraction of $m_{\text {top }}^{\text {pole }}$ from normalised differential cross-sections [2]. Figure (a) shows the distribution of the transverse momentum of the electron-muon system observed in data compared to MC simulation events. For this variable, figure (b) shows the ratio of the predicted differential cross-section at NLO in production and decay and the measured one with experimental and theoretical uncertainties. Finally, figure (c) shows $m_{\mathrm{top}}^{\text {pole }}$ extracted from various variables and from a simultaneous fit to all of them. All results are given as a function of the proton PDF used in the extraction.

\section{Measurement of the top quark mass}

The results listed in Tab. 1 are obtained in the dilepton channel [3], the lepton+jets channel [4] and finally, in the all-jets channel [5]. In general, measurements of $m_{\text {top }}$ receive large uncertainties induced by the jet energy scale (JES) uncertainties, evaluated for all jets and by the relative $b$-to-light-jet energy scale (bJES) uncertainty that only relates to $b$-quark initiated jets. Those uncertainties account for a large fraction of the experimental uncertainties listed in Tab. 1. Aiming at the most precise value of $m_{\text {top }}$ in the combination of results, and not in individual channels, methods are preferred that reduce the uncertainties, while retaining or even better, reducing the correlations $(\rho)$ of the various estimators. This is because in this case large improvements over the 


\begin{tabular}{rccccccc} 
Channel $(\sqrt{s})$ & Value & Statistics & Modelling & Background & Experimental & Total & Ref. \\
\hline Dilepton $(8 \mathrm{TeV})$ & 172.99 & 0.41 & $0.35 \pm 0.09$ & $0.08 \pm 0.01$ & $0.64 \pm 0.04$ & 0.84 & {$[3]$} \\
Lepton+jets $(7 \mathrm{TeV})$ & 172.33 & 0.75 & $0.53 \pm 0.11$ & $0.31 \pm 0.00$ & $0.82 \pm 0.08$ & 1.27 & {$[4]$} \\
All-jets $(8 \mathrm{TeV})$ & 173.72 & 0.55 & $0.70 \pm 0.16$ & 0.19 & $0.71 \pm 0.04$ & 1.15 & {$[5]$} \\
\hline
\end{tabular}

Table 1: The measured values of $m_{\text {top }}$ are given together with the statistical uncertainties, systematic uncertainties subdivided into modelling, background related and experimental uncertainties and the total uncertainty (in $[\mathrm{GeV}]$ ). For each systematic uncertainty listed, the first value corresponds to the uncertainty in $m_{\text {top }}$, and the second (if available) to the statistical precision of this uncertainty.

most precise result, i.e. the knowledge in $m_{\text {top }}$ without combination, are obtained [6]. In ATLAS, two main paths to mitigate the jet energy scale induced uncertainties in $m_{\text {top }}$ are followed. The first is to use an $m_{\text {top }}$ sensitive observable that is stabilised against JES variations. The second is to use additional information in the data sensitive to global changes in the jet energy scales. This information is then used for protecting the $m_{\text {top }}$ sensitive observable against global shifts, by absorbing them into global jet energy scale factors, named JSF and bJSF.

The analysis in the all-jets channel uses the first path pioneered for the lepton+jets channel in Ref. [7]. The analysis exploits the ratio of the three-jet mass and the two-jet mass $\left(R_{3 / 2}\right)$ as $m_{\text {top }}$ sensitive variable, shown in Fig. 2(a). Since the JES induced variations in the jet energies at the same time apply to the jets in the numerator and denominator, most of the effects cancel. This cancellation results in a reduced uncertainty in $m_{\text {top }}$ from this source than otherwise would be obtained when directly using the reconstructed top quark mass $\left(m_{\mathrm{top}}^{\text {reco }}\right)$ for measuring $m_{\mathrm{top}}$.

The measurement in the lepton+jets channel uses the second path. Together with $m_{\mathrm{top}}^{\text {reco }}$, shown in Fig. 2(b), two additional distributions are exploited. These are the reconstructed invariant mass of the $W$-boson and the ratio of transverse momenta of the two $b$-jets and the two light jets assigned to the hadronic $W$-boson decay. The first distribution is sensitive to the JES. The second distribution is sensitive to the bJES, while, as for $R_{3 / 2}$, the JES dependence mostly cancels in this ratio of transverse momenta. The price to pay is additional contributions to the statistical uncertainty in $m_{\text {top }}$ caused by fitting to more distributions. However, for sufficiently large data samples, this loss in statistical precision is more than compensated for by the strong reduction in the respective systematic uncertainty in $m_{\text {top }}$, induced by the jet energy scale uncertainty. This situation is realised in Ref. [4]. Not only does this achieve a smaller total uncertainty $(\sigma)$ in $m_{\text {top }}$ for this decay channel, it also transforms a significant part of the systematic uncertainty into a statistical uncertainty. This has two positive consequences. Firstly, this statistical component to the uncertainty will naturally be reduced by including more data. Secondly, given that the results in $m_{\text {top }}$ for the various decay channels are uncorrelated with respect to their statistical uncertainties, it also potentially helps in reducing the estimator correlations.

The missing hadronic $W$-boson decay in the dilepton channel prevents the use of the above methods. However, the clean final state in this channel results in high selection efficiency at high signal purity. The low background is barely visible for the distribution of the average invariant mass of the charged-lepton- $b$-jet systems $\left(m_{l b}^{\text {reco }}\right)$ shown in Fig. 2(c). This would result in a very unbalanced composition of statistical and systematic uncertainty, i.e. indicating a non-optimised analysis. Consequently, the large data sample is used to reduce the total uncertainty by trading 


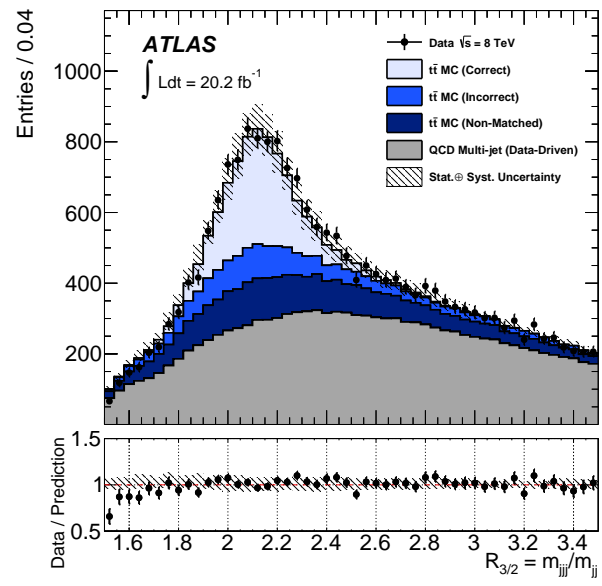

(a) $m_{\text {top }}$ sensitive distribution, all-jets channel

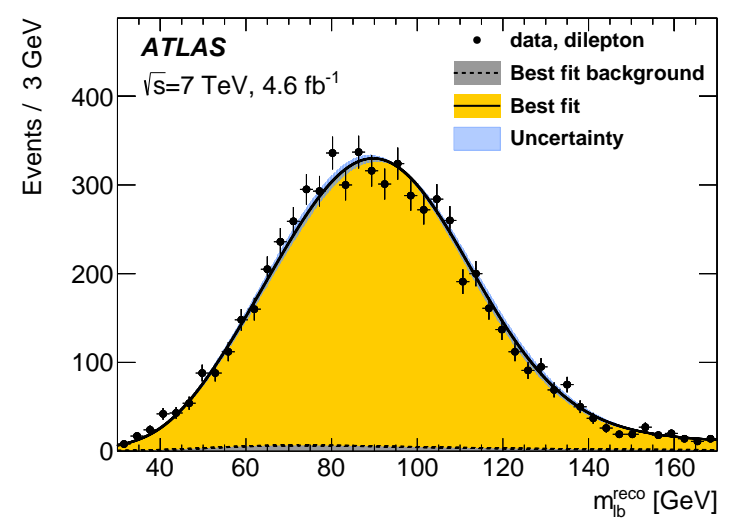

(c) $m_{\text {top }}$ sensitive distribution, dilepton channel

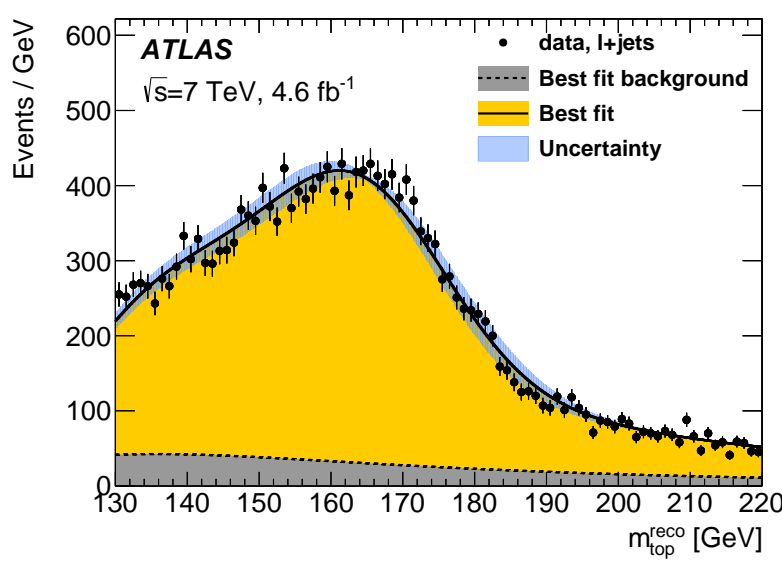

(b) $m_{\text {top }}$ sensitive distribution, lepton+jets channel

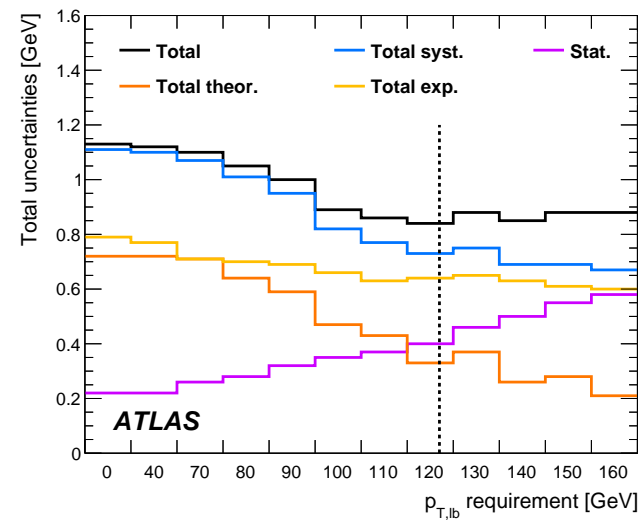

(d) Uncertainty optimisation, dilepton channel

Figure 2: Measurements of $m_{\text {top }}$ from template fits. Figure (a) shows the ratio of the three-jet invariant mass to the two-jet invariant mass $\left(R_{3 / 2}\right)$ in the all-jets channel [5]. Figure (b) shows the reconstructed top quark mass $\left(m_{\mathrm{top}}^{\text {reco }}\right)$ in the lepton+jets channel [4]. Figure $(\mathrm{c}, \mathrm{d})$ are obtained from the two charged-lepton- $b$-jet systems in the dilepton channel [3]. Figure (c) shows the average reconstructed invariant mass $\left(m_{l b}^{\text {reco }}\right)$ of the two systems. Finally, figure (d) shows the total uncertainty $(\sigma)$ in $m_{\text {top }}$ for the dilepton channel as a function of the minimum requirement on the average transverse momenta of the two systems $\left(p_{\mathrm{T}, l b}\right)$.

statistical for systematic precision based on additional phase space restrictions. The result of this optimisation [3] as a function of the average transverse momentum of the two charged-lepton$b$-jet systems $\left(p_{\mathrm{T}, l b}\right)$ is shown in Fig. 2(d). Compared to the result without this requirement, a reduction of $26 \%$ in the uncertainty in $m_{\text {top }}$ is achieved. This requirement removes $74 \%$ of the original events, thereby resulting in an $86 \%$ increase in the statistical uncertainty, i.e. no gain in resolution is achieved by this phase space restriction. Again, given a significant part of the systematic uncertainty is transformed into a statistical uncertainty, this potentially helps in reducing the estimator correlations. 


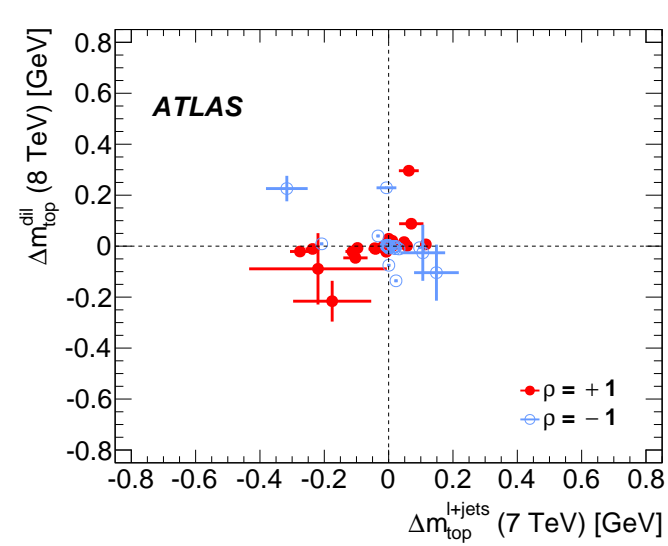

(a) Pairwise correlation - lepton+jets versus dilepton

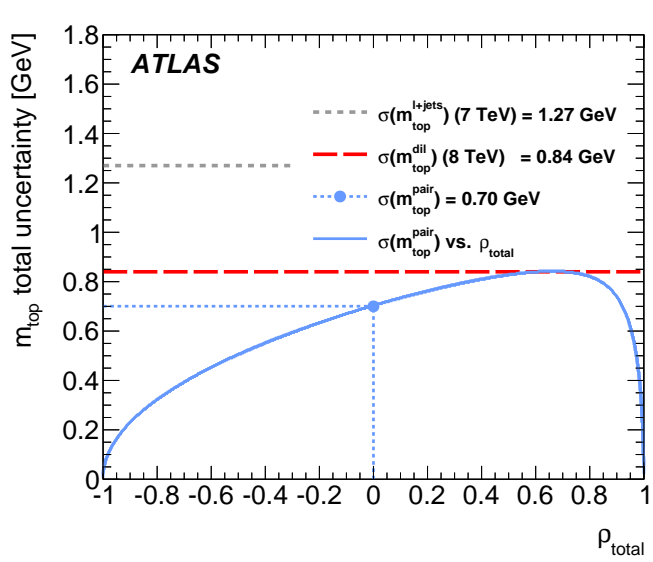

(b) Combination of the two most precise results

Figure 3: Figure (a) shows the differences in the top quark mass $\left(\Delta m_{\text {top }}\right)$ when simultaneously varying a pair of measurements for the subcomponents of a systematic uncertainty using the pairs indicated [3]. The red full points indicate the fully correlated cases, the blue open points the anti-correlated ones. See text for further details. Figure (b) shows the uncertainty in the combined result of the combination of the most precise pair of results as a function of the estimator correlation $\rho_{\text {total }}$. The blue point corresponds to the actual estimator correlation [3].

\section{Combination of measurements}

The combination is performed using the best linear unbiased estimate (BLUE) method $[8,6]$ in a $\mathrm{C}++$ implementation described in Ref. [9]. The BLUE method combines measurements based on a linear combination of the inputs. The coefficients (BLUE weights) are determined via the minimisation of the total variance of the combined result. They can be used to construct measures for the importance of a given single measurement in the combination [6]. The measured values of $m_{\text {top }}$, the list of uncertainty components and the correlations of the estimators for each uncertainty component have to be provided. The first two are given in the respective publications, while the estimator correlations need to be obtained. As developed in Ref. [4], for the ATLAS combination of measurements of $m_{\text {top }}$, the correlations are evaluated for each source of systematic uncertainty as displayed in Fig. 3(a) for the most precise pair of estimators. Each point corresponds to one subcomponent of the systematic uncertainty in $m_{\text {top }}$, obtained by varying this pair of measurements for this subcomponents. Using MC simulated events, pseudo-experiments are constructed of the size of the ATLAS data sample and $m_{\text {top }}$ is fitted to those. The point is located at the mean values of the observed shifts in the top quark mass $\left(\Delta m_{\mathrm{top}}\right)$ for both analyses. This location is calculated from the pseudo-experiments. The cross indicates the statistical precisions in the systematic uncertainties as given by the precision of the pseudo-experiments. Uncertainties for which the estimators are correlated are located in the first and third quadrant and are shown as red full points, the anti-correlated cases, located in the remaining two quadrants, are displayed by open blue points. The ATLAS combination is dominated by the two input results shown in Fig. 3(b). At the quoted precision, the combination of just this pair achieves the identical total uncertainty as the full combination. Finally, the 2016 combined ATLAS result for the top quark 
mass is: $m_{\text {top }}=172.84 \pm 0.34$ (stat.) \pm 0.61 (syst.) $\mathrm{GeV}=172.84 \pm 0.70 \mathrm{GeV}$, with a precision of $0.4 \%$. Evaluating statistical uncertainties for each systematic uncertainty in $m_{\text {top }}$ allows for performing stability tests on the combination without ad-hoc assignments of variations in estimator correlations. For each combination, the size of the uncertainty and the correlation are newly evaluated, based on random variations of each systematic uncertainty within its statistical precision. As a result, both the combined value and the corresponding uncertainty are stable to within $0.03 \mathrm{GeV}$.

\section{Conclusions}

An extraction of the top quark pole mass at NLO is presented. The result is obtained from normalised differential cross-sections in the $t \bar{t} \rightarrow$ dilepton channel. The uncertainty in the result of: $m_{\mathrm{top}}^{\text {pole }}=173.2 \pm 0.9$ (stat.) \pm 0.8 (syst.) \pm 1.2 (theo.) $\mathrm{GeV}=173.2 \pm 1.6 \mathrm{GeV}$, is dominated by systematic effects, mainly by scale variations.

In addition, measurements of $m_{\text {top }}$ are discussed, which are based on the template method in the three $t \bar{t}$ decay channels, $t \bar{t} \rightarrow$ dilepton, $t \bar{t} \rightarrow$ lepton+jets and $t \bar{t} \rightarrow$ all-jets. The uncertainties in the two most precise results in $m_{\text {top }}$ have been reduced using methods paying attention to the correlations of the estimators, enabling a significant gain in precision in $m_{\text {top }}$ in their combination. The 2016 combined ATLAS result for the top quark mass is:

$$
m_{\text {top }}=172.84 \pm 0.34 \text { (stat.) } \pm 0.61 \text { (syst.) } \mathrm{GeV}=172.84 \pm 0.70 \mathrm{GeV}
$$

with a precision of $0.4 \%$. The statistical precision in the total uncertainty is $0.03 \mathrm{GeV}$.

\section{References}

[1] ATLAS Collaboration, The ATLAS Experiment at the CERN Large Hadron Collider, JINST 3 (2008) S08003.

[2] ATLAS Collaboration, Measurement of lepton differential distributions and the top quark mass in $t \bar{t}$ production in pp collisions at $\sqrt{s}=8 \mathrm{TeV}$ with the ATLAS detector, ATLAS-CONF-2017-044, 2017, [https://cds.cern.ch/record/2273850].

[3] ATLAS Collaboration, Measurement of the top quark mass in the $t \bar{t} \rightarrow$ dilepton channel from $\sqrt{s}=8 \mathrm{TeV}$ ATLAS data, Phys. Lett. B 761 (2016) 350, [1606.02179].

[4] ATLAS Collaboration, Measurement of the top quark mass in the t $\bar{t} \rightarrow$ lepton + jets and $t \bar{t} \rightarrow$ dilepton channels using $\sqrt{s}=7$ TeV ATLAS data, Eur. Phys. J. C 75 (2015) 330, [1503.05427].

[5] ATLAS Collaboration, Top-quark mass measurement in the all-hadronic $t \bar{t}$ decay channel at $\sqrt{s}=8 \mathrm{TeV}$ with the ATLAS detector, 1702.07546.

[6] R. Nisius, On the combination of correlated estimates of a physics observable, Eur. Phys. J. C 74 (2014) 3004, [1402.4016].

[7] ATLAS Collaboration, Measurement of the top quark mass with the template method in the $t \bar{t} \rightarrow$ lepton + jets channel using ATLAS data, Eur. Phys. J. C 72 (2012) 2046, [1203.5755].

[8] A. Valassi, Combining correlated measurements of several different quantities, Nucl. Instr. and Meth. A $\mathbf{5 0 0}(2003) 391$.

[9] R. Nisius, BLUE: a software package to combine correlated estimates of physics observables within ROOT using the Best Linear Unbiased Estimate method, 2015, [http: / / b l ue . hep forge org]. 